cartographic events

\section{EVENTS CALENDAR}

1992

January 18: 1990 Census Data User Conference, New Orleans, LA. Contact: U.S. Census Bureau's Data User Services Division, User Training, Washington, DC 20244; (301) 763-1510.

February 4-7: ICOGRAPHICS '92, Milan, Italy. Contact: World Computer Graphics Association, Ste. 399, 2033 M St., NW, Washington, DC 20036. (202) 775-9556; fax: (202) $775-8122$.

February 9-14: GIS '92, 6th Annual Symposium, Working Smarter, Geographic Information Systems in Forestry, Environment and Natural Resources Management, Vancouver, British Columbia, Canada. Contact: GIS '92 Symposium Office, 720-845 Cambie Street, Vancouver, British Columbia, Canada V6B 4Z9, (604) 688-0188; fax: (604) 688-1573.

February 11-14: MICAD '92, Paris, France. Contact: World Computer Graphics Association, 2033 M St., NW Ste. 399, Washington, DC 20036. (202) 775-9556; fax: (202) 775-8122.

February 25-27: Mapping Awareness '92. Conference and Exhibition, Olympia 2, London. Contact: Lindsey Deakin, Blenheim Online, Blenheim House, Ash Hill Drive, Pinner, Middlesex, HA5 2AE, UK telephone: 0818684466.

March 17-20: SIG-GIS '92, Paris, France. Contact: World Computer Graphics Association, Ste. 399, 2033 M St., NW , Washington, DC 20036. (202) 775-9556; fax: (202) $775-8122$.

March 22-28: ACSM/ASPRS Annual Convention, Albuquer-

que, NM. Contact: ACSM, 5410 Grosvenor Lane, Bethesda, MD 20814, (301) 493-0200.

June 2-6: Canadian Cartographic Association and Carto-Quebec Joint Annual Conference, Montreal, Canada. Contact: Jacqueline M. Anderson, Department of Geography, Concordia University, 1455 de Maisonneuve Blvd. W., Montreal, Quebec H3G 1M8, (514) 848-2052; email JAHFH10@VAX2.CONCORDIA.CA; fax: (514) 848-3494, Attention:

Geography Department.

June 15-18: Computer Vision \& Pattern Recognition, Chancellor Hotel and Convention Center, Champaign, IL. Contact: Azriel Rosenfeld, e-mail: ar@alv.umd.edu

Summer: Fifth International Symposium on Spatial Data Handling, USA. Contact: Prof. Duane F. Marble, Department of Geography, The Ohio State University, Columbus, $\mathrm{OH} 43210$, (614) 292-2250, telex: (650) 218-4975 MCI.

August 2-14: 17th International Society for Photogrammetry and Remote Sensing Congress, Washington, DC. Contact: 17th ISPRS Congress Secretariat, Box 7147, Reston, VA 22091.

August 9-16: 27th International Geographical Congress, Washington, DC. Contact: Anthony de Sousa, 27th IGC, 17th and M Sts. NW, Washington, DC 20036, (202) 828-6688.

October 14-17: North American Cartographic Information Society XII, St. Paul, MN. Contact: Jeff Patton, Department of Geography, University of North Carolina at Greensboro, Greensboro, NC 27412; (919) 334-5388.
November 6-12: GIS/LIS 1992 Annual Conference and Exposition and ACSM/ASPRS Fall Convention, San Jose, CA. Contact: ACSM, 5410 Grosvenor Lane, Bethesda, MD 20814, (301) 493-0200.

1993

February 15-18: ACSM/ASPRS Annual Convention, New Orleans, Louisiana. Contact: ACSM, 5410 Grosvenor Lane, Bethesda, MD 20814, (301) 493-0200.

\section{NACIS news}

\section{NACIS OFFICERS}

President: Jack L. Dodd, Tennessee Valley Authority, 1101 Market St., HB 1A, Chattanooga, TN 374022801; (615) 751-5404.

Vice President: Jeff Patton, Department of Geography, University of North CarolinaGreensboro, Greensboro, NC 27412; (919) 334-5388.

Secretary: Craig Remington, Geography Department, University of Alabama, Tuscaloosa, AL 35487; (205) 348-1536.

Treasurer: Edward J. Hall, 410 McGilvrey Hall, Kent State University, Kent, OH 44240-0001; (216) 672-2017.

Past President: James F. Fryman, Department of Geography, University of Northern Iowa, Cedar Falls, IA 50613; (319) 273-6245.

\section{NACIS EXECUTIVE OFFICER}

Chris Baruth, NACIS, American Geographic Society Collection, P.O. 399, Milwaukee, WI 53201; (800) 558-8993 or (414) 229-6282

\section{BOARD OF DIRECTORS}

James R. Anderson, Jr., FREAC, 361 Bellamy Building, Florida State 